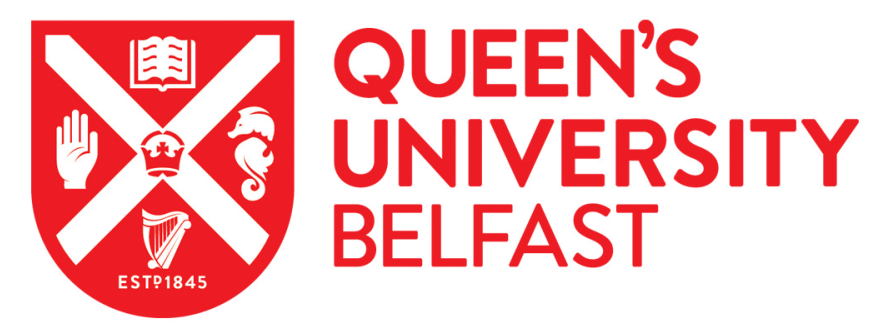

\title{
In situ electron holography of the dynamic magnetic field emanating from a hard-disk drive writer
}

\author{
Einsle, J. F., Gatel, C., Masseboeuf, A., Cours, R., Bashir, M. A., Gubbins, M., Bowman, R. M., \& Snoeck, E. \\ (2015). In situ electron holography of the dynamic magnetic field emanating from a hard-disk drive writer. Nano \\ Research, 8(4), 1241-1249. https://doi.org/10.1007/s12274-014-0610-0
}

\section{Published in:}

Nano Research

Document Version:

Peer reviewed version

Queen's University Belfast - Research Portal:

Link to publication record in Queen's University Belfast Research Portal

Publisher rights

The final publication will be available at Springer via http://dx.doi.org/10.1007/s12274-014-0610-0

\section{General rights}

Copyright for the publications made accessible via the Queen's University Belfast Research Portal is retained by the author(s) and / or other copyright owners and it is a condition of accessing these publications that users recognise and abide by the legal requirements associated with these rights.

Take down policy

The Research Portal is Queen's institutional repository that provides access to Queen's research output. Every effort has been made to ensure that content in the Research Portal does not infringe any person's rights, or applicable UK laws. If you discover content in the Research Portal that you believe breaches copyright or violates any law, please contact openaccess@qub.ac.uk. 


\title{
In situ electron holography of the dynamic magnetic field emanating from a hard-disk drive writer
}

\author{
Joshua F. Einsle ${ }^{1}$, Christophe Gatel ${ }^{2,3}(\bowtie)$, Aurélien Masseboeuf ${ }^{2}$, Robin Cours ${ }^{2}$, Muhammad A. \\ Bashir $^{4}$, Mark Gubbins ${ }^{4}$, Robert M. Bowman ${ }^{1}$, Etienne Snoeck ${ }^{2}$
}

Nano Res., Just Accepted Manuscript • DOI: 10.1007/s12274-014-0610-0

http://www.thenanoresearch.com on October 162014

C Tsinghua University Press 2014

\section{Just Accepted}

This is a "Just Accepted" manuscript, which has been examined by the peer-review process and has been accepted for publication. A "Just Accepted" manuscript is published online shortly after its acceptance, which is prior to technical editing and formatting and author proofing. Tsinghua University Press (TUP) provides "Just Accepted" as an optional and free service which allows authors to make their results available to the research community as soon as possible after acceptance. After a manuscript has been technically edited and formatted, it will be removed from the "Just Accepted" Web site and published as an ASAP article. Please note that technical editing may introduce minor changes to the manuscript text and/or graphics which may affect the content, and all legal disclaimers that apply to the journal pertain. In no event shall TUP be held responsible for errors or consequences arising from the use of any information contained in these "Just Accepted" manuscripts. To cite this manuscript please use its Digital Object Identifier (DOI®), which is identical for all formats of publication. 


\section{Template for Preparation of Manuscripts for Nano Research}

This template is to be used for preparing manuscripts for submission to Nano Research. Use of this template will save time in the review and production processes and will expedite publication. However, use of the template is not a requirement of submission. Do not modify the template in any way (delete spaces, modify font size/line height, etc.). If you need more detailed information about the preparation and submission of a manuscript to Nano Research, please see the latest version of the Instructions for Authors at http://www.thenanoresearch.com/.

\section{TABLE OF CONTENTS (TOC)}

Authors are required to submit a graphic entry for the Table of Contents (TOC) in conjunction with the manuscript title. This graphic should capture the readers' attention and give readers a visual impression of the essence of the paper. Labels, formulae, or numbers within the graphic must be legible at publication size. Tables or spectra are not acceptable. Color graphics are highly encouraged. The resolution of the figure should be at least $600 \mathrm{dpi}$. The size should be at least $50 \mathrm{~mm} \times 80 \mathrm{~mm}$ with a rectangular shape (ideally, the ratio of height to width should be less than 1 and larger than 5/8). One to two sentences should be written below the figure to summarize the paper. To create the TOC, please insert your image in the template box below. Fonts, size, and spaces should not be changed.

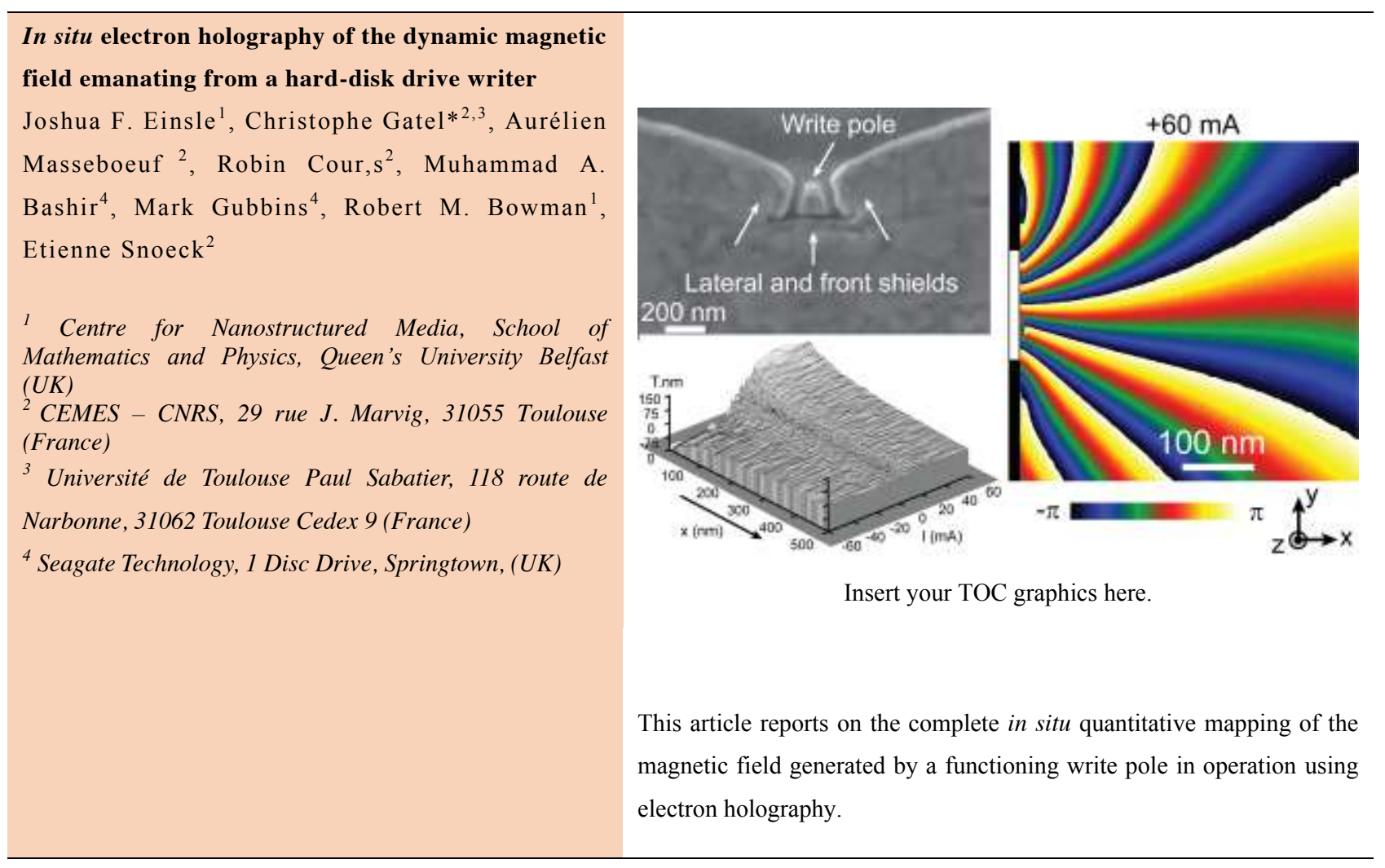

Provide the authors' webside if possible.

C. Gatel, http://www.cemes.fr/ 


\title{
In situ electron holography of the dynamic magnetic field emanating from a hard-disk drive writer
}

\author{
Joshua F. Einsle ${ }^{1}$, Christophe Gatel ${ }^{2,3}(\triangle)$, Aurélien Masseboeuf ${ }^{2}$, Robin Cours ${ }^{2}$, \\ Muhammad A. Bashir ${ }^{4}$, Mark Gubbins ${ }^{4}$, Robert M. Bowman ${ }^{1}$, Etienne Snoeck ${ }^{2}$ \\ ${ }^{1}$ Centre for Nanostructured Media, School of Mathematics and Physics, Queen's University Belfast (UK) \\ ${ }^{2}$ CEMES - CNRS, 29 rue J. Marvig, 31055 Toulouse (France) \\ ${ }^{3}$ Université de Toulouse Paul Sabatier, 118 route de Narbonne, 31062 Toulouse Cedex 9 (France) \\ ${ }^{4}$ Seagate Technology, 1 Disc Drive, Springtown, (UK)
}

Received: day month year Revised: day month year Accepted: day month year (automatically inserted by the publisher)

(C) Tsinghua University Press and Springer-Verlag Berlin Heidelberg 2014

\section{KEYWORDS}

Electron transmission microscopy, off-axis electron holography, in situ, magnetic field, magnetic recording, hard disk write pole

\begin{abstract}
The proliferation of mobile devices in society accessing data via the 'cloud' is imposing a dramatic increase in the amount of information to be stored on hard disk drives (HDD) used in servers. Forecasts are that areal densities will need to increase by as much as $35 \%$ compound per annum and by 2020 cloud storage capacity will be around 7 zettabytes corresponding to areal densities of $2 \mathrm{~Tb} / \mathrm{in}^{2}$. This requires increased performance from the magnetic pole of the electromagnetic writer in the read/write head in the HDD. Current state-of-art writing is undertaken by morphologically complex magnetic pole of sub 100 $\mathrm{nm}$ dimensions, in an environment of engineered magnetic shields and it needs to deliver strong directional magnetic field to areas on the recording media around $50 \mathrm{~nm} \times 13 \mathrm{~nm}$. This points to the need for a method to perform direct quantitative measurements of the magnetic field generated by the write pole at the nanometer scale. Here we report on the complete in situ quantitative mapping of the magnetic field generated by a functioning write pole in operation using electron holography. Opportunistically, it points the way towards a new nanoscale magnetic field source to further develop in situ Transmission Electron Microscopy.
\end{abstract}

Address correspondence to Christophe Gatel, gatel@cemes.fr 


\section{Introduction}

The read/write head is the small active part of the slider component located at the end of the actuator arm inside a HDD. A schematic drawing of a slider is shown in

Fig. 1a. In Fig. 1b is a SEM image of the read/write parts that face the media with a zoom of the write pole region. The yellow arrows illustrate the direction of travel of the media beneath the write pole. The writing/erasing magnetic field is generated by the current flowing in the coils intended to behave linearly with the applied current without hysteresis when reversing the current to allow for the fast, accurate writing and erasing of data [1] (a cross section of the read/write element is presented in Fig. S1 and S2 in the Electronic Supplementary Material (ESM)on which the yoke and return poles of the writer electromagnet and the drive coils are identified). To precisely tailor the profile of emanating magnetic field, the current carrying coils and bulk magnetic material of the writer are shielded from the disk media. This also insures that the magnetic environment experienced by the media is dominated by the write pole and not from other surfaces of this three dimensional electromagnetic device. These shields are composed of soft magnetic materials to intensify the magnetic field gradients coming out of the write pole and are shown in the zoom Fig. 1b.

Current understanding about the behaviour, shape and strength of the magnetic field generated by this nanoscale electromagnet arises from indirect methods. Either the size and shape of the written magnetic bits are measured on the magnetic media [2] or numerical simulations are performed to visualize the fields emanating from the pole. With continued downscaling of the write pole to critical dimensions numerical approaches will become essential but computationally complex. Additionally, the development of new technologies like Heat Assisted Magnetic Recording (HAMR) $[3,4]$ which combines an optical field to
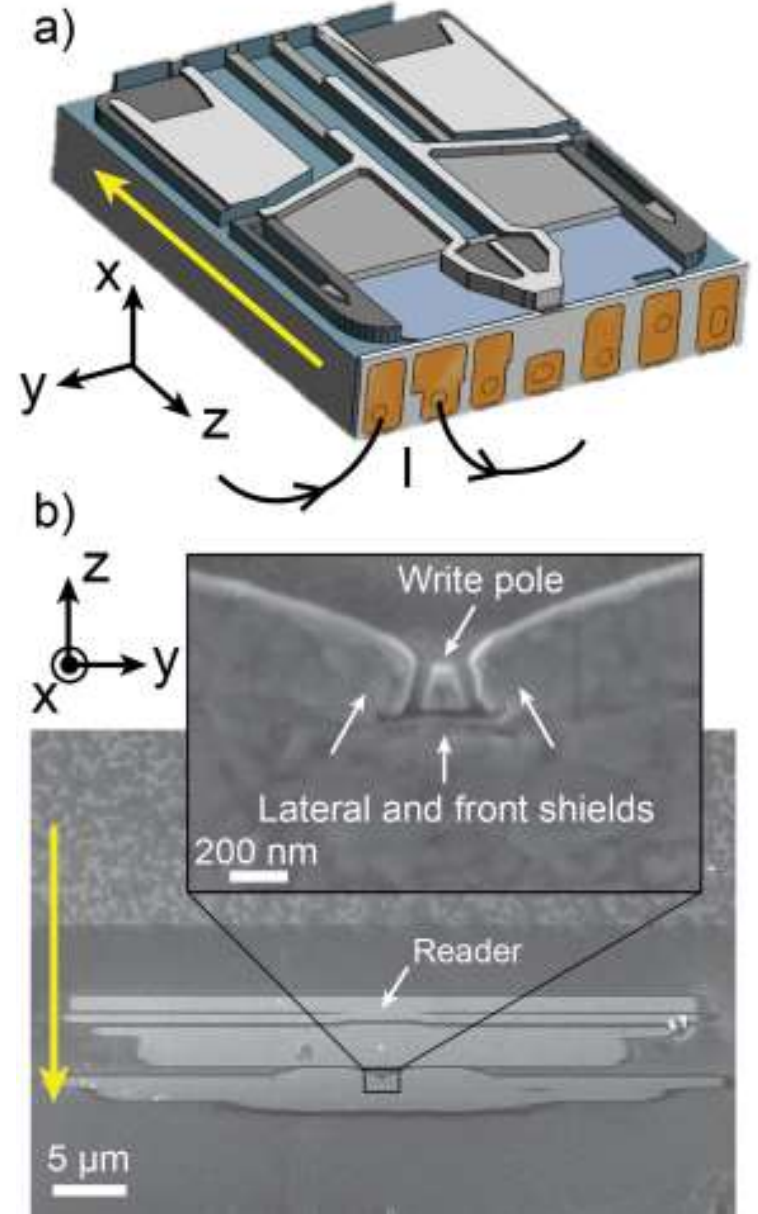

Figure 1 a) Schematic illustration of the HDD slider (I corresponds to the injected current), b) High magnification SEM image of the read/write parts with a zoom of the write pole inset. The yellow arrows indicate the flying direction of the magnetic media and the electron path for TEM experiments. The xy plane will correspond to the studied area in the following figures while the $\mathrm{z}$-axis is parallel to the electron path.

reduce the media coercivity with the writing/erasing magnetic field will make magnetic write tests even more difficult. A direct approach for the visualization and quantification of the magnetic field produced by a write pole would be of significant technological benefit for the characterization of HDD read/write heads performance. In this study, we have developed such a tool, by combining the powerful TEM technique of off-axis electron holography $(\mathrm{EH})$ with in situ electrical biasing to actively vary the magnetic 
field produced by a fully operational HDD write pole. Electron Holography (EH) is an efficient interferometric TEM method enabling the quantitative mapping of local electrostatic and magnetic fields with a resolution of few nanometres over a field of view as large as few microns [5-9]. For this particular study, we have developed a new set-up allowing for the first time to quantitatively map the magnetic fields generated by the writer as a function of applied electrical currents up to $\pm 60 \mathrm{~mA}$. The resulting quantified magnetic field maps demonstrate the key features required in magnetic recording, namely a highly directional magnetic field with low angular spread.

Further, while we are able to tune the applied current in series of steady state steps, this dynamic fluxing of the magnetic field paves way for two innovative applications in EH. The writer and its wiring circuitry are designed to operate to $\mathrm{GHz}$ frequencies; it therefore opens up the potential for the acquisition of quantitative information of the magnetic induction generated by the writer as a function of the applied current frequency. Then, of more general interest, it points to a new development of new in situ EH TEM experiments whereby a nanoscale magnetic field source can be incorporated into sample stage assemblies for in situ magnetic experiments of wider interest.

\section{Experimental}

The write pole has been an extensively studied device due to its importance in the operation of HDDs. Along with other techniques, EH has been used to study thin section lamellae of the write pole [7,8] and demonstrates how magnetic domains form under various externally applied magnetic fields. These basic experiments do not, however, give information about the generation of a magnetic field by the write pole itself in operating conditions when electric current is sent into the coils. Recently, in situ EH approaches have been attempted to characterize the write pole device [9]. While Goto et al. demonstrate that a field can be imaged in situ they did not fully isolate and characterize the magnetic field of the write pole in operational conditions. Presenting only partial magnetic information with the writer still imbedded in the shield material, the magnetic signal resulting of the current flowing in the coils cannot be separate from the one due to the shields neither from additional artefacts originating from electrostatic potential. We have developed a novel strategy that allows imaging an operational write pole in situ in a TEM in normal conditions, to obtain the complete local hysteresis loop on each point of a large area and quantitatively separate the magnetic signals originating solely from the write pole and those of the shield material, while dynamically changing the applied current through the write coils.

As outlined the difficulty in developing a method for directly imaging the write pole's magnetic fields lies in keeping the necessary electrical circuitry undamaged to drive the writer, while making it fit in a TEM stage. In this work, we achieve this through reducing the overall size of a HDD slider from $1.2 \mathrm{~mm}$ down to $30 \mu \mathrm{m}$ by mechanically polishing while keeping intact the electrical contacts pads and coils used by the device. An additional issue is to provide a scheme to locate the plane in which the write pole resides, because in this $\mathrm{EH}$ experiment the electron beam passes adjacent to the face of the write pole. To do so, Focussed Ion Beam Induced Deposition was used to grow a pair of fiducial carbon pillars on each side of the write pole prior to TEM investigation. The thinned device is then wire bonded to an in situ electrical biasing holder and transferred to the microscope (sample preparation details are given in $S I$ ).

The in situ TEM experiment was performed in Lorentz mode on a Cs-corrected Tecnai F20 microscope operating at $200 \mathrm{kV}$. A Möllensted biprism, positioned parallel to the surface of the slider at a distance of about $600 \mathrm{~nm}$, is used to create the interference pattern between part of the 
electron beam interacting with the generated magnetic induction field in front of the write pole and part of the beam that passes far from it through an almost field-free region, so-called "reference beam" (a low magnification image of the whole area is given in the SI). The analysis of the interference pattern (i.e. the electron hologram) allows extraction of the phase shift between the reference beam and the one that has interacted with the magnetic induction.

In our experiment, the electron phase shift (r) is only related to the magnetic induction through the Aharanov-Bohm relation [10,13]:

$$
\phi(\mathbf{r})=-\frac{e}{\hbar} \iint B_{\perp}(\mathbf{r}, z) d r d z
$$

where $\mathbf{r}=(x, y)$ represents the position vector in the plane perpendicular to the electron path $(x$ and $y$ directions), $e$ the elementary charge, $B_{\perp}$ the component of the magnetic induction in the plane perpendicular to $\mathbf{r}$ and to the beam direction $z$. The measured phase shift corresponds to a projection and an integration of all in-plane magnetic field contributions along the electron path. Numerical simulation are generally unavoidable to take into account the two dimensional projection of a three dimensional field.

\section{Results and discussion}

\subsection{Magnetic phase shift as function of current}

Applying a current on the device generates a magnetic field that can be directly visualized studying the phase image calculated in-line from the hologram [14]. After determining the precise location of the write pole and adjusting carefully the orientation of its exposed surface parallel to the electron beam, we fully characterized the generated magnetic induction by collecting two series of 44 holograms. A first sequence was acquired with the applied current varying from $-60 \mathrm{~mA}$ to $+60 \mathrm{~mA}$, and a second series reverses this sequence by running the current from $+60 \mathrm{~mA}$ to $-60 \mathrm{~mA}$ to effectively perform a hysteresis loop measurements. Phase shift images obtained with currents of -60 $\mathrm{mA},-10 \mathrm{~mA}, 0 \mathrm{~mA}, 10 \mathrm{~mA}, 60 \mathrm{~mA}$ are reported in the left column of Fig. 2. The projected magnetic field is parallel to the isophase lines. Considering that $B_{\perp}=\left(B_{x}, B_{y}\right)$, the $x$ and $y$ components of the magnetic induction integrated over the beam path can then be extracted calculating respectively the $y$ and $x$ derivatives of the phase shift image (after unwrapping to remove the phase jumps) following the equations:

$$
\begin{aligned}
& \int B_{x}(\mathbf{r}) d z=-\frac{\hbar}{e} \frac{d}{d y} \phi(\mathbf{r}) \\
& \int B_{y}(\mathbf{r}) d z=\frac{\hbar}{e} \frac{d}{d x} \phi(\mathbf{r})
\end{aligned}
$$

$\int B_{y}(\mathbf{r}) d z$ and $\int B_{y}(\mathbf{r}) d z$ are mapped using Eq.

$2 \mathrm{a}$ and $2 \mathrm{~b}$ and reported in the two columns on the right side of Fig. 2 for the five corresponding currents. The phase images and magnetic field components are symmetric with respect to the " $x$ " axis for applied current of opposite sign. For $0 \mathrm{~mA}$ applied current the magnetic flux is only between the shields located on each side of the pole indicating that the integrated $B_{x}$ component of the magnetic induction is zero for the direction perpendicular to the write pole.

Beside these two dimensional maps of the magnetic induction generated by the write pole in operation, the $\mathrm{EH}$ experiment allows for the quantitative determination of the induction field at any point of the studied area and for each value of the applied D.C. current. It then permits extraction of local hysteresis loops of the two components of the magnetic induction. As an illustration, we extracted four hysteresis loops of the $B_{x}$ and $B_{y}$ components averaged over a $(5 \times 5) \mathrm{nm}^{2}$ region at four different 


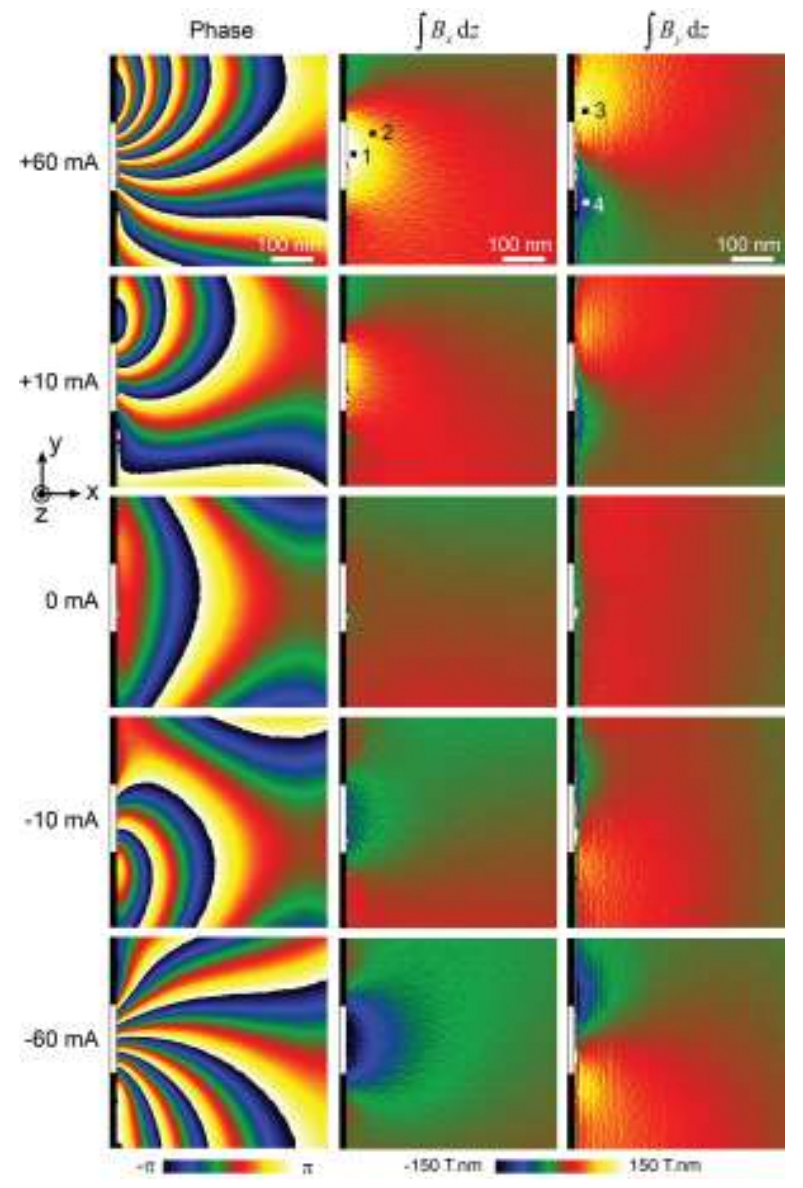

Figure 2 Left column: Experimental phase image of the magnetic flux recorded in front of the write pole for an applied current of $+60 \mathrm{~mA},+10 \mathrm{~mA}, 0 \mathrm{~mA},-10 \mathrm{~mA}$ and $-60 \mathrm{~mA}$. Middle column: Corresponding $\mathrm{B}_{\mathrm{x}}$ components map integrated over the beam path. Right column: Corresponding $\mathrm{B}_{\mathrm{y}}$ components map integrated over the beam path. The white rectangle on the middle left side of each image is the write pole location.

positions in front of the device (noted 1, 2, 3, 4 in the first $B_{x}$ and $B_{y}$ images of Fig. 2) for current varying from $+60 \mathrm{~mA}$ to $-60 \mathrm{~mA}$ then from $-60 \mathrm{~mA}$ to $+60 \mathrm{~mA}$. These local hysteresis loops are reported in Figs. $3 \mathrm{a}$ and $3 \mathrm{~b}$ for $B_{x}$ and $B_{y}$ respectively. The two $B x$ hysteresis loops in Fig. 3a evidence that the magnetic saturation is reached for an applied current of about $25 \mathrm{~mA}$. They also indicate the absence of remnant and coercive fields that both are expected for a HDD read/write device. This results also evidences the induction field reduction when moving away from the central part of the pole i.e. when moving from position (1) located at $5 \mathrm{~nm}$ in front of pole to position (2) displaced by $50 \mathrm{~nm}$ both in $x$ and $y$ directions (This magnetic field decrease is also evidenced on Fig. S5).

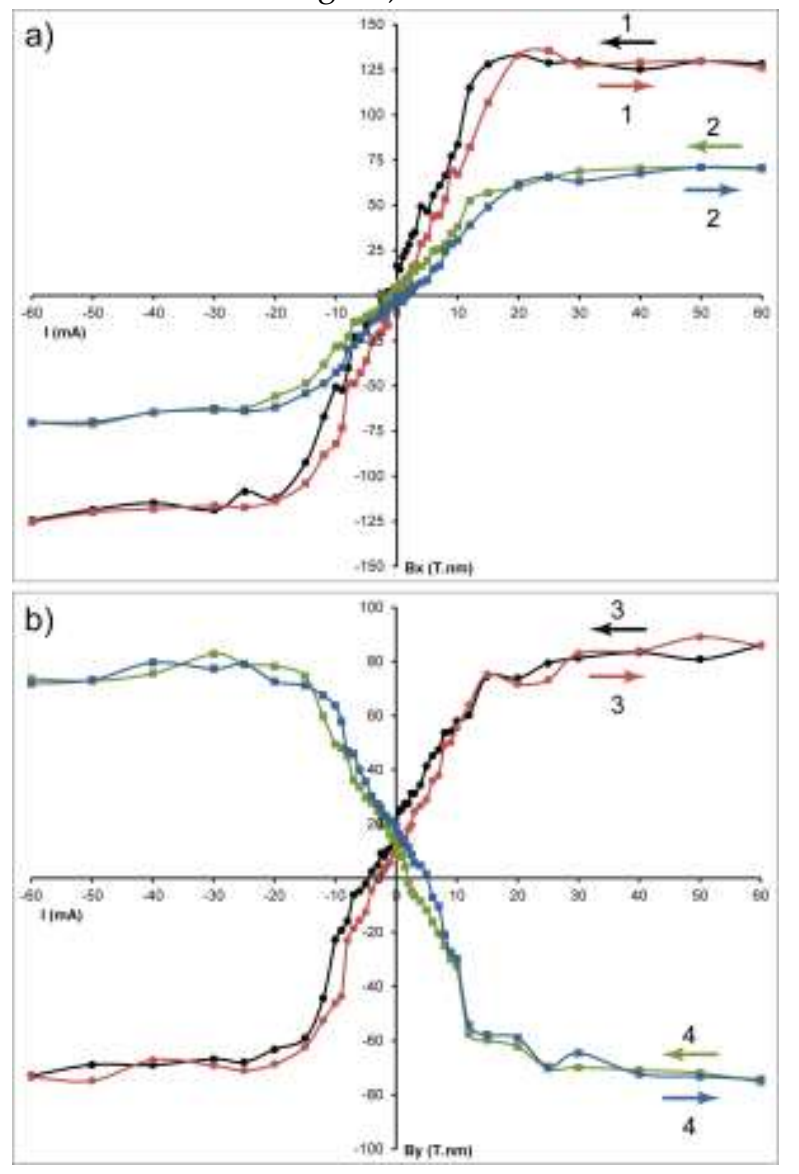

Figure 3 a) Hysteresis loops of the $B_{x}$ component extracted from Fig. 2 (middle column) at position (1) and (2), b) Hysteresis loops of the $\mathrm{B}_{\mathrm{y}}$ component extracted from Fig. 2 (right column) at position (3) and (4).

The two $B_{y}$ hysteresis loops reported in Fig. $3 \mathrm{~b}$ for two symmetrical positions (3) and (4) on each side of the pole (at position $x=20 \mathrm{~nm}$ and $y=+/-100 \mathrm{~nm}$ ) show that the magnetic induction remains symmetric with respect to the write pole. In addition, these $B_{y}$ hysteresis loops indicate the appearance of a constant magnetic field for zero applied current. This magnetic field is due to the lateral magnetic shields adjacent to the write pole. 
The total magnetic induction in front of the write pole is therefore the superimposition of the constant magnetic induction created by the shields and the one generated by the current sent into the writer coils. Changing the polarity of the applied current results in reversing the direction of the induced magnetic field while the shield magnetic induction remains constant. Therefore, the half-sum of two phase-images obtained with reversal current gives the magnetic contribution of the shields while their half difference results in the phase contribution of the writer only. Fig. 4 illustrates the results of these calculations. The images on the top show the magnetic induction created by the writer only for applied currents of $0 \mathrm{~mA},+10 \mathrm{~mA}$ and $+60 \mathrm{~mA}$ while the ones at the bottom of the image are the magnetic induction of the shield. The later remains constant and indicates that the shield produces an almost flat and constant magnetic field running between the shields from one side of the write pole to the other. The gradient of the shield induction is perpendicular to the write pole one and much lower.

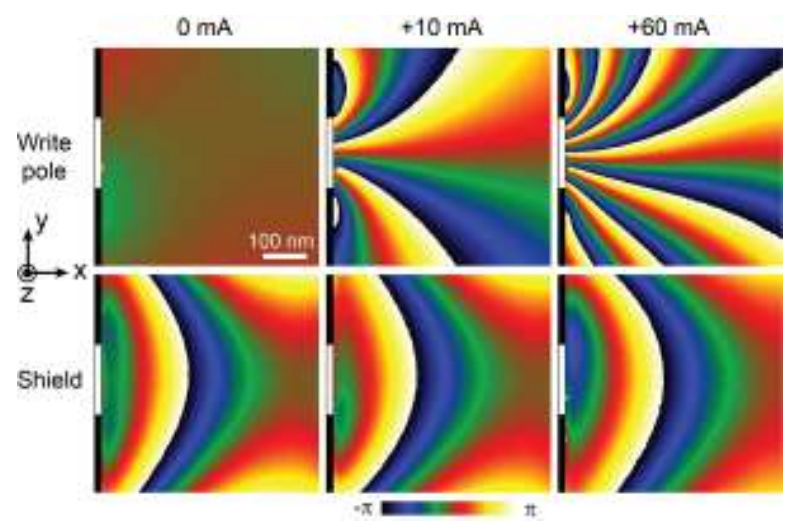

Figure 4 Upper row: Experimental images of the magnetic flux generated by the write pole for applied currents of $0 \mathrm{~mA},+10$ $\mathrm{mA}$ and $+60 \mathrm{~mA}$, Bottom row: Experimental images of the magnetic flux due to the shield which remains constant.

\subsection{Micromagnetic Simulations}

Such quantitative measurements of the magnetic induction should be confirmed by numerical simulation to ensure that the region where the reference beam has been selected is indeed field free, or contains a negligible field, and does not create artefacts in the final phase image. We use the finite element package Magpar [15] to simulate the entire write pole system using the dimensions of the write pole extracted from SEM images. The standard bulk values of the magnetic constants of the various materials comprising the write pole system are used (writer, shields, etc.). We ensure that the device model and its materials are saturated by the application of an initial current of $+40 \mathrm{~mA}$ before extracting steady state information. We then generate the magnetic flux spreading in a three dimensional $(1 \mu \mathrm{m}, 1.5 \mu \mathrm{m}, 0.8 \mu \mathrm{m})$ region in front of the whole device for an applied current of +40 $\mathrm{mA}$ (an image of the simulation box is reported in SI Fig. S3 in the ESM).

The resulting magnetic induction flux is used to calculate the phase shift of the electron beam passing across the box. The resulting phase image is reported in Fig. 5a. We then calculated the difference between the simulated phase of the electron beam that has passed within a $500 \mathrm{~nm}$ wide region close to the write pole («object wave area » in Fig. 5a) and the simulated phase of the electron beam that has passed in $500 \mathrm{~nm}$ wide region which is not perfectly field free located $500 \mathrm{~nm}$ far from it ( reference wave area » in Fig. 5a).

The resulting phase image is reported in Fig. $5 \mathrm{~b}$ and presents a very good agreement with the experimental one at the same injected current (Fig. $5 c)$. We can note that the modification of the simulated phase due to the weak field in the reference wave area is negligible. A more quantitative comparison can be achieved drawing the simulated (including or nit the reference wave contribution) and experimental induction profiles from the $B_{x}$ component at $40 \mathrm{~mA}$ (Fig. 6a and 6b) along two directions: one along the " $x$ " direction at position $y=0$ (Fig. 6c), the other along the " $y$ "

(19) TNIVERGIYYPRES TS Springer I www.editorialmanager.com/nare/default.asp 
direction at position $x=5 \mathrm{~nm}$ from the writer surface corresponding to the position of the media in operation (Fig. 6d). The simulated profiles fit very well with the experimental ones and confirm the negligible effect arising from the remaining field of the reference area. This result supports both the model we use for the device and the $\mathrm{EH}$ procedure. It also confirms that the magnetic induction generated by the write pole is as expected when saturating it, for the materials and geometrical considerations.
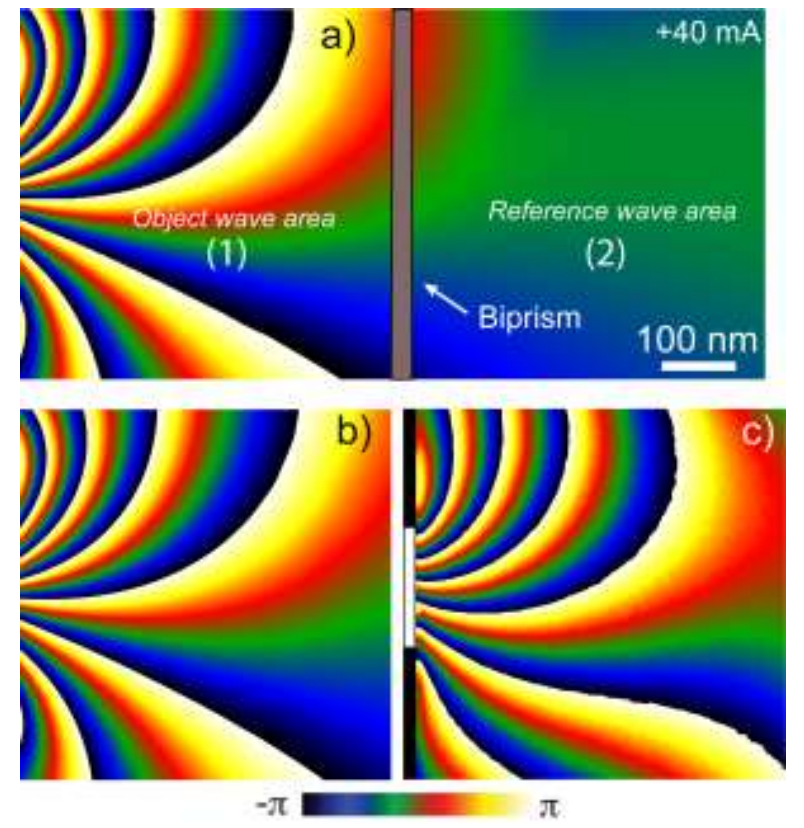

Figure 5

a) Simulated phase of the electron beam after its interaction with the device magnetic induction over a $500 \mathrm{~nm} \times 1 \mu \mathrm{m}$ box and projected along the beam path. The biprism is located in the middle of the image and creates interference between the regions 1 and 2 of the electron beam passing on each side of it. The simulation has been realized for an applied current of +40 $\mathrm{mA}$.

b) Simulated phase image resulting on the difference of the phases in region 1 and 2 taking into account the influence of the remaining magnetic induction in the reference beam. The modification arising from the remaining field of the reference area is negligible.

c) Experimental phase image at $+40 \mathrm{~mA}$. We can note the very nice agreement with the simulation image in $b$ ).

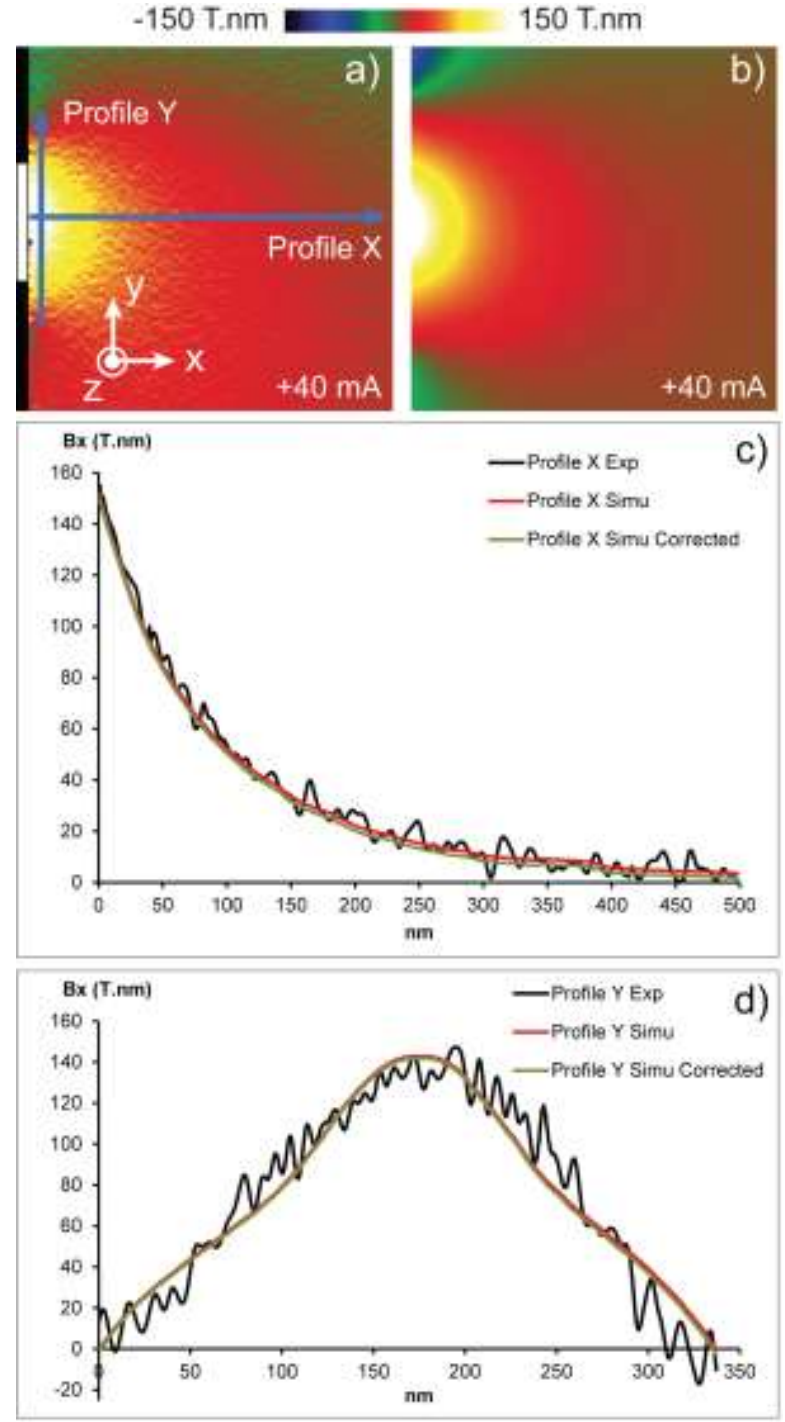

Figure 6

a) Experimental image of the $B_{x}$ component of the magnetic induction for a $+40 \mathrm{~mA}$ applied current.

b) Simulated image of the $B_{x}$ component of the magnetic induction for a $+40 \mathrm{~mA}$ applied current.

c) Experimental (black) profile of the $B_{x}$ component extracted along the " $\mathrm{x}$ " direction at position $\mathrm{y}=0$ (horizontal blue arrow on a)). Simulated profiles in red (without reference area correction) and green (with correction).

d) Experimental (black) profile of the $B_{x}$ component extracted along the " $\mathrm{y}$ " direction at position $\mathrm{x}=5 \mathrm{~nm}$ from the writer surface (vertical blue arrow on a)). Simulated profiles in red (without reference area correction) and green (with correction). 


\subsection{Magnetic induction mapping}

We have shown that $\mathrm{EH}$ allows the quantitative mapping of the magnetic induction in front of the device and therefore is appropriate to analyse its capability to focus the flux to a recording media. In addition, the write pole contribution can be perfectly isolated. These in situ $\mathrm{EH}$ experiments allows building quantitative 3D data cubes $B_{x}(x, y, I)$ and $B_{y}(x, y, I)$ of the local magnetic induction components relevant to characterize the magnetic field generated by the write pole only at various $(x$, $y$ ) positions in front of it (Fig. 7a). The component of the hysteresis loop perpendicular to the device, $B_{x}$, is of particular interest as it is the part of the induction that writes magnetic bits on the media. A clear visualization of $B_{x}$ can be obtained plotting a series of $B_{x}(I)$ hysteresis loops extracted from the data cube $B_{x}(x, y, I)$ at position $y=0$, in front of the write pole piece (noted (1) on the Fig. 7a), and for increasing distance from it (i.e. as function of " $x$ "). This surface plot is reported in Fig. $7 \mathrm{~b}$ together with the $B_{x}(x, y, I)$ data cube (Fig. 7a).
It shows that the symmetry of the hysteretic behavior remains with a decreasing magnitude versus the distance from the write pole down to $0 \mathrm{~T}$ as the sampling position goes out beyond $400 \mathrm{~nm}$. A similar surface plot can be extracted to evidence how focused the magnetic induction is $5 \mathrm{~nm}$ in front of the writer, which is approximately the distance between the read/write head and the media in normal operation and is reported in Fig. 7c (extracted from position noted (2) on the Fig. 7a). Further manipulations of the $B_{x}$ and $B_{y}$ data cubes allow quantifying the magnetic flux spreading by calculating $\left|\tan ^{-1}\right| B_{y} / B_{x}||$ for a given current. It results in an image that exhibits the magnetic induction spread from the write pole source to the vacuum. In Fig. 8a is reported the angular spread of the magnetic flux observed for a $+60 \mathrm{~mA}$ applied current. A quantitative measurement of the magnetic flux divergence can be extracted at the position where the magnetic media is flying $5 \mathrm{~nm}$ in front of the pole. For $+60 \mathrm{~mA}$ applied current the
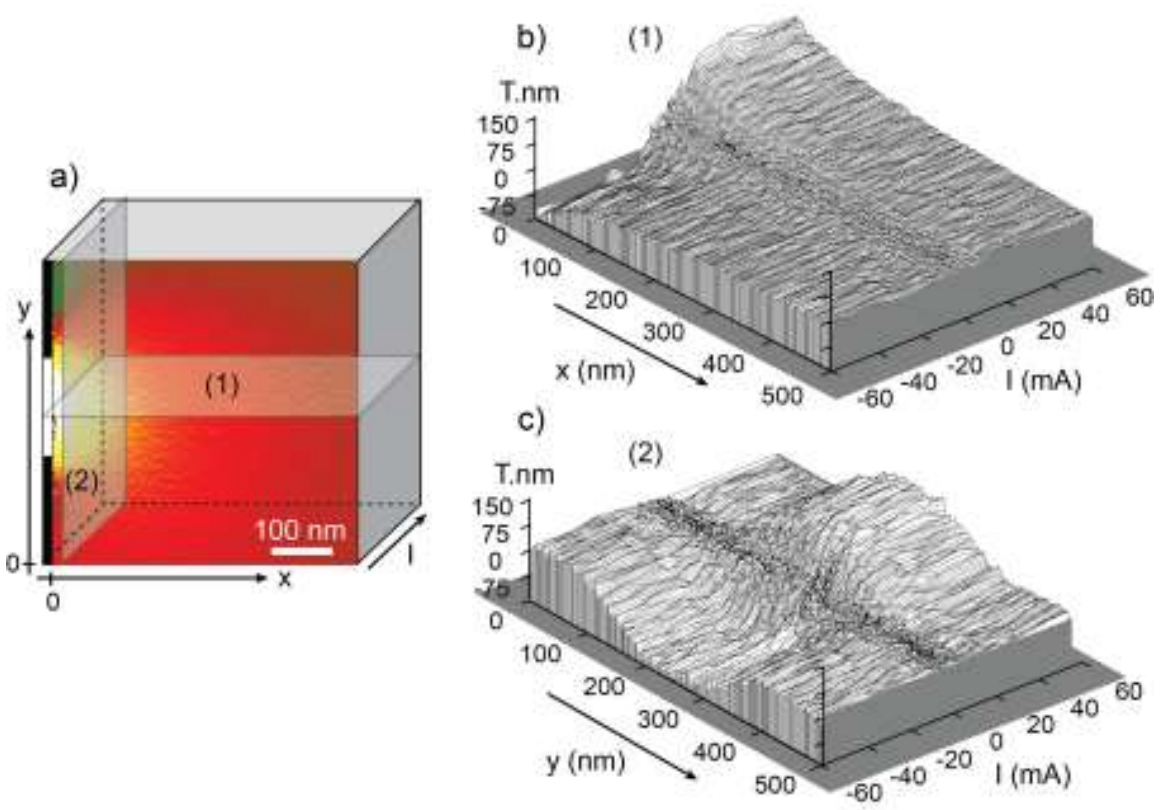

Figure 7 a) The experimental $B_{x}(x, y, I)$ data cube of the write pole contribution, b) $B_{x}(I)$ surface plot of the hysteresis loops extracted from a) at $y=0$ for increasing distance from the write pole (plane (1) in Fig 7a), c) $B_{x}(I)$ surface plot of the hysteresis loops extracted from a) as function of the lateral " $y$ " positions and at a distance $x=5 \mathrm{~nm}$ from the pole (plane (2) in Fig 7a). 

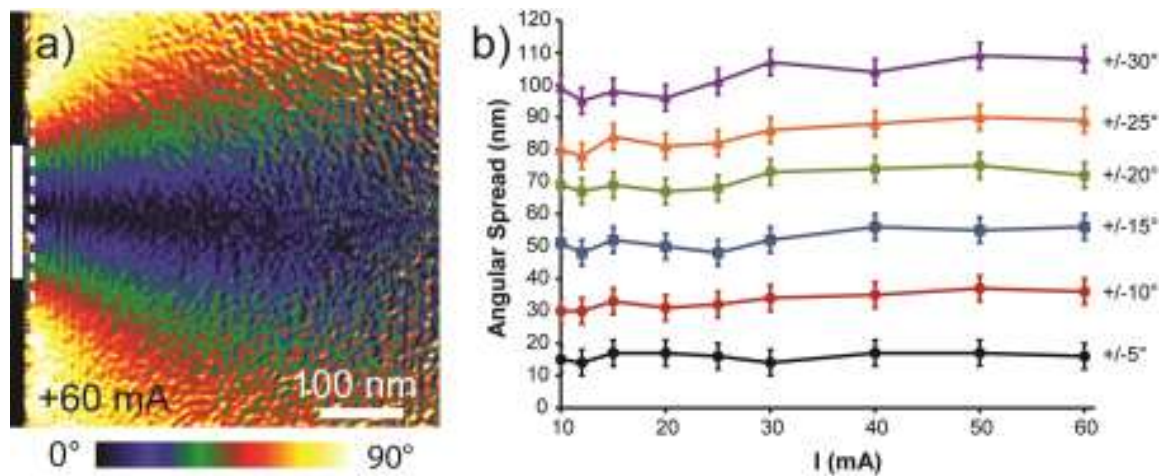

Figure 8 a) Angular spread of the magnetic flux observed for an applied current of $+60 \mathrm{~mA}, \mathrm{~b}$ ) Variation of the radiated region width at $5 \mathrm{~nm}$ in front of the pole (dashed line in (a) as a function of the applied current and for various flux divergences.

magnetic flux spreading over $\Delta \theta=10^{\circ}$ angle (noted $+/-5^{\circ}$ on Fig. $8 \mathrm{~b}$ is radiating over a $15 \mathrm{~nm}$ wide region at $5 \mathrm{~nm}$ from the pole. We plot in Fig. 8a the variation of the radiated regions located at $5 \mathrm{~nm}$ in front of the pole as a function of the applied current and for various flux divergences. This demonstrates how the lateral shields are efficient in focusing the field onto the media for current varying between 10 $\mathrm{mA}$ and $60 \mathrm{~mA}$ and how the flux drops off laterally (to prevent interference across adjacent recorded tracks in the media). From the HDD application point of view this illustrates that only the media directly under the write pole will experience any significant magnetic interaction for writing/erasure processes.

\section{Conclusions}

In summary we have developed a new in situ TEM process allowing the quantitative measurement of the magnetic field generated by a HDD writer in operation at the nanometer scale. The combination of off-axis electron holography with micromagnetic simulations allows us to quantify the full magnetic response of the write pole to various applied currents. We demonstrate that there is no remnant field along the perpendicular direction to the surface of the write pole when no current is applied. We have been able to confidently determine the magnetic field in saturation conditions and to measure the local hysteresis loop for any point of the studied region. We show how to separate the different contributions of the shield and the write pole and characterize the angular spread of the magnetic field as a function of current. We have therefore demonstrated the power of holography to characterize active magnetic systems and provided baseline device characterization important for design of next generation write poles. As the control circuitry of the writer are designed to accommodate time varying signals up to the $\mathrm{GHz}$ range, further in situ electron holography experiments are now feasible to analyze the magnetic induction damping as function of the applied current frequency used in data storage.

In addition, this work has laid the foundation for the development of a new type of in situ magnetic instrumentation for TEM. A HDD write device could well be used to create a local magnetic source for fundamental magnetic studies in situ in TEM. Since the magnetic field coming off the pole tip is located in the in-plane direction, various magnetic nanomaterials could be placed into a tunable magnetic field generated by the write pole and their local magnetic response studied in situ. This would open up new methods for studying systems like data storage media under realistic conditions. Additionally, more exotic systems can now be explored in more detail such as magnetic nanoparticles, magnetic tunnel junctions or artificial spin ices [16,17]. 


\section{Acknowledgements}

The authors acknowledge the European Union under the Seventh Framework Programme under a contract for an Integrated Infrastructure Initiative Reference 312483-ESTEEM2 and the French National Research Agency under the "Investissement d'Avenir" program reference No. ANR-10-EQPX-38-01". This work is supported by the French national project EMMA (ANR12 BS10 013 01).

Electronic Supplementary Material: Supplementary material giving some details about the sample preparation, the electron holography quantification and the micromagnetic simulation is available in the online version of this article at http://dx.doi.org/10.1007/s12274-***_****-*

(automatically inserted by the publisher).

\section{References}

[1] Richter, H. J. The transition from longitudinal to perpendicular recording. J. Phys. D. Appl. Phys. 2007, 40, R149-R177.

[2] Manalis S., Babcock K., Massie J., Elings V. and Dugas V. Submicron studies of recording media using thin film magnetic scanning probes Appl. Phys. Lett. 1995, 66, 2585 .

[3] Stipe, B. C. et al. Magnetic recording at $1.5 \mathrm{~Pb} \mathrm{~m}-2$ using an integrated plasmonic antenna. Nat. Photonics 2010, 4, 484-488.

[4] Challener, W. A. et al. Heat-assisted magnetic recording by a near-field transducer with efficient optical energy transfer. Nat. Photonics 2009, 3, 220-224.

[5] Thomas, J. M., Simpson, E. T., Kasama, T. \& Dunin-Borkowski, R. E. Electron holography for the study of magnetic nanomaterials. Acc. Chem. Res. 2008, $41,665-74$

[6] Masseboeuf, A., Marty, A., Bayle-Guillemaud, P., Gatel, C. \& Snoeck, E. Quantitative observation of magnetic flux distribution in new magnetic films for future high density recording media. Nano Lett. 2009, 9, 2803-6.
[7] Kim, J. J. et al. Magnetic domain observation in writer pole tip for perpendicular recording head by electron holography. Appl. Phys. Lett. 2008, 92, 162501.

[8] Hirata, K., Ishida, Y., Akashi, T., Shindo, D. \& Tonomura, A. Electron holography study of magnetization behavior in the writer pole of a perpendicular magnetic recording head by a $1 \mathrm{MV}$ transmission electron microscope. $J$. Electron Microsc. (Tokyo) 2012, 61, 305-8.

[9] Goto, T. et al. Electron holography of magnetic field generated by a magnetic recording head. Microscopy 2013, 62, 383-9.

[10] Ehrenberg, W. \& Siday, R. E. The Refractive Index in Electron Optics and the Principles of Dynamics. Proc. Phys. Soc. Sect. B 1949, 62, 8-21.

[11] Aharonov, Y. \& Bohm, D. Significance of Electromagnetic Potentials in the Quantum Theory. Phys. Rev. 1959, 115, 485-491.

[12] Aharonov, Y. \& Bohm, D. Further Considerations on Electromagnetic Potentials in the Quantum Theory. Phys. Rev. 1961, 123, 1511-1524.

[13] Tonomura, A. et al. Observation of Aharonov-Bohm Effect by Electron Holography. Phys. Rev. Lett. 1982, 48, $1443-1446$

[14] Snoeck E. and Gatel C. In Magnetic Mapping Using Electron Holography in Transmission Electron Microscopy in Micro-nanoelectronics ed. A. Claverie, Editeur : ISTE Ltd and John Wiley \& Sons Inc 2012

[15] Scholz, W. et al. Scalable parallel micromagnetic solvers for magnetic nanostructures. Comput. Mater. Sci. 2003, $28,366-383$

[16] Ladak, S., Read, D. E., Perkins, G. K., Cohen, L. F. \& Branford, W. R. Direct observation of magnetic monopole defects in an artificial spin-ice system. Nat. Phys. 2010, 6, 359-363.

[17] Zhang, S. et al. Crystallites of magnetic charges in artificial spin ice. Nature 2013, 500, 553-557. 


\title{
Electronic Supplementary Material
}

\section{In situ electron holography of the dynamic magnetic field emanating from a hard-disk drive writer}

\author{
Bashir $^{4}$, Mark Gubbins ${ }^{4}$, Robert M. Bowman ${ }^{1}$, Etienne Snoeck ${ }^{2}$ \\ ${ }^{1}$ Centre for Nanostructured Media, School of Mathematics and Physics, Queen's University Belfast (UK) \\ ${ }^{2}$ CEMES - CNRS, 29 rue J. Marvig, 31055 Toulouse (France) \\ ${ }^{3}$ Université de Toulouse Paul Sabatier, 118 route de Narbonne, 31062 Toulouse Cedex 9 (France) \\ ${ }^{4}$ Seagate Technology, 1 Disc Drive, Springtown, (UK)
}

Joshua F. Einsle ${ }^{1}$, Christophe Gatel ${ }^{2,3}(\bowtie)$, Aurélien Masseboeuf ${ }^{2}$, Robin Cours ${ }^{2}$, Muhammad A.

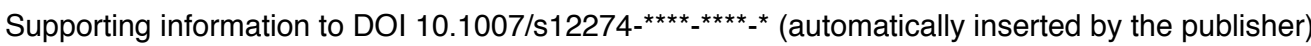

\section{Sample preparation}

The slider was firstly mechanical polished down to $30 \mu \mathrm{m}$ along the $\mathrm{z}$ direction (see Fig. S1) using a tripod polishing system. The remaining bar of the slider containing the writing head as well as the contact pads was then mounted on a traditional TEM sample grid (insulating) laying along the $(x, y)$ plane, contacts at the top (see Fig. S2.c). The device was then put into the FIB for growing two small carbon pillars ( $2 \mu \mathrm{m}$ wide, $5 \mu \mathrm{m}$ long) by Ion Beam Induced Deposition on both sides ( $y$ direction) of the write pole ( $5 \mu \mathrm{m}$ away on each side - see Fig. S2.a and b). The device was then mounted into a home-made sample holder allowing 2 vacuum feedthroughs towards the outside (Fig. S2.c). Wedge bonding was then use to connect the contact pads of the slider toward the dedicated contacts of the sample holder (Fig. S2.c enlargement).

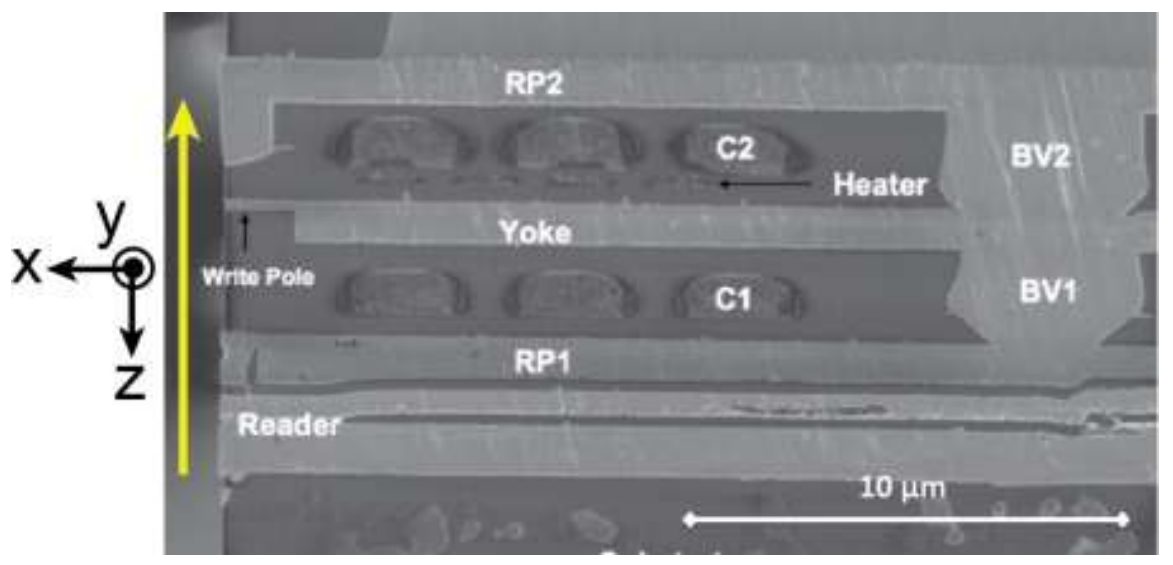

Figure S1 Cross-section SEM image of the read/write head. The magnetic shields and the write pole are evidenced. The yellow arrow indicates the flying direction of the magnetic media and the electron path for TEM experiments. This sectional view corresponds to the $x z$ plane; the studied area is the xy plane. 


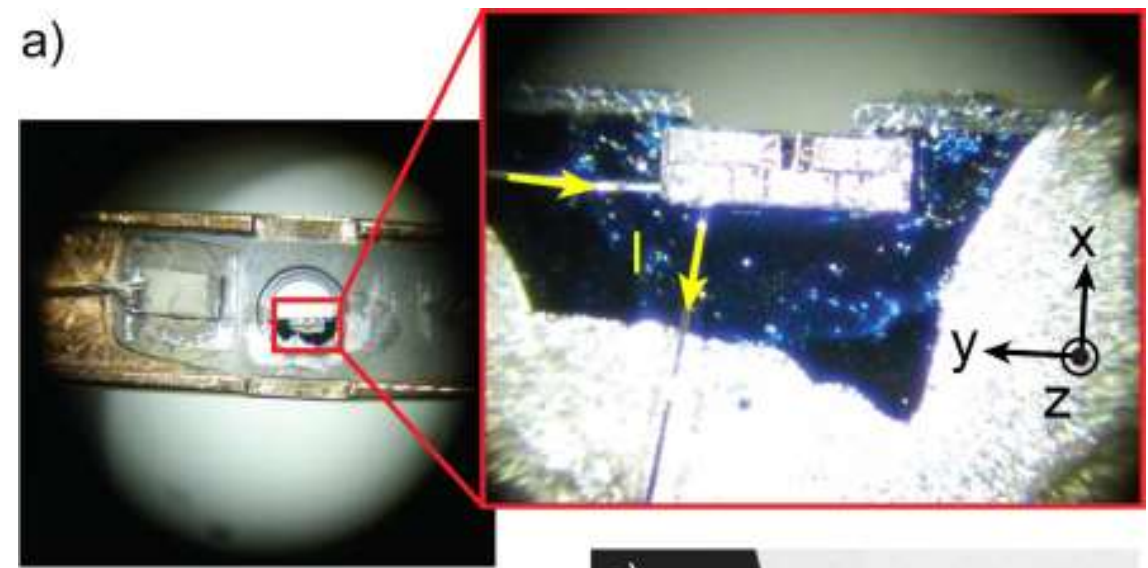

\section{b)}
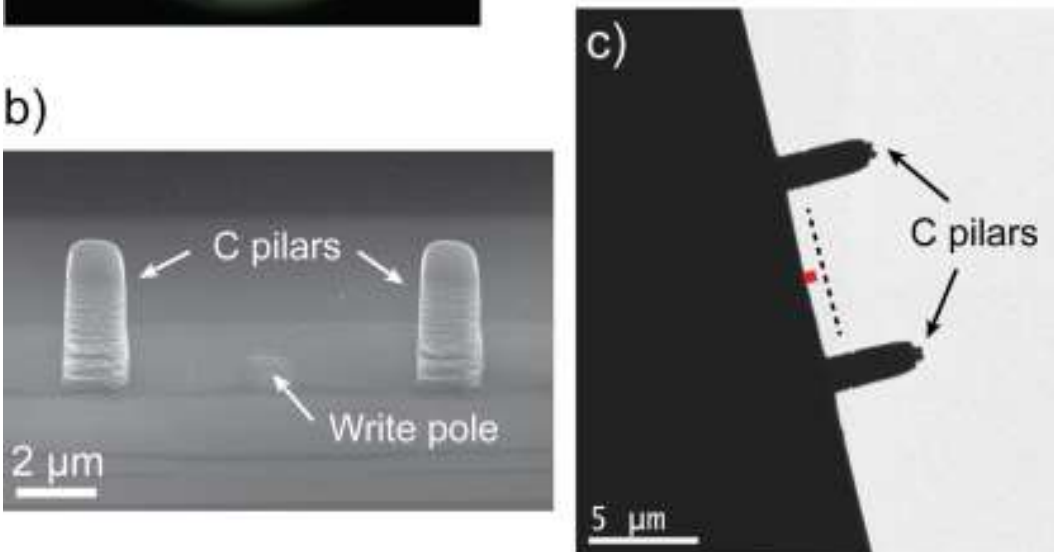

\section{Figure S2}

a) Carbon nanopillars deposited by FIBID allowing the location of the write pole for the TEM experiment.

b) TEM image of the write pole with the carbon pillars. The red square and the dashed line represent respectively the studied area in front of the write pole and the position of the biprism.

c) Image of the thin device positioned in the biasing stage with the wire bounding.

\section{Electron holography}

The phase shift of an electron beam that has interacted with an electromagnetic field is sensitive both to the electrostatic potential " $V$ " and to the components of the magnetic vector potential " $A z$ " and integrated along the beam path.

The measured phase shift is derived from the Aharonov-Bohm expression giving the phase shift of a charged particles interacting with an electrostatic potentials and a magnetic vector potential [S1].

$$
\begin{aligned}
& \phi(\mathbf{r})=C_{E} \int V(\mathbf{r}, z) d z-\frac{e}{\hbar} \int A_{z}(\mathbf{r}, z) d z \\
& \phi(\mathbf{r})=C_{E} \int V(\mathbf{r}, z) d z-\frac{e}{\hbar} \iint B_{\perp}(\mathbf{r}, z) d r d z(\mathrm{~S} 2)
\end{aligned}
$$

where $C_{E}=\frac{2 \pi}{\lambda} \frac{E_{k}+E_{0}}{E_{k}\left(E_{k}+2 E_{0}\right)}$

(9.9) TNIVERGIYYPRES TS Springer I www.editorialmanager.com/nare/default.asp 
- $z$ is the direction of the incident beam,

- $\mathbf{r}$ is the position vector in the plane perpendicular to beam direction,

- $A z$ the component of the magnetic vector potential " $\mathbf{A}$ " along the beam direction,

- $B_{\perp}$ the component of the magnetic induction perpendicular to both $\mathbf{r}$ and $z$,

- $V$ the electrostatic potential

- $\lambda$ the electron wavelength

- $E_{0}$ the rest mass energy of the electrons

- $E_{\kappa}$ the kinetic energy of the electrons

In the vacuum and without the presence of any electrostatic field, which is the case in our study on the write head, only the second term of Eq. S2 is acting [S2].

The phase image is calculated from the electron hologram performing its Fourier Transform (FT) and selecting one of the two side band reflections. The selecting reflection is masked and re-centered before to compute the Inverse Fourier Transform (FT-1). This leads to a complex image whose amplitude image and phase image can be separated.

The resulting phase image is then $\phi(\mathbf{r})=-\frac{e}{\hbar} \iint B_{\perp}(\mathbf{r}, z) d r d z$

where $\mathbf{r}=(x, y)$ represents the position vector in the plane perpendicular to the electron path $(x$ and $y$ directions), $e$ the elementary charge, $B_{\perp}$ the component of the magnetic induction in the plane perpendicular to $\mathbf{r}$ and to the beam direction $z$. The measured phase shift corresponds to a projection and an integration of all in-plane magnetic field contributions along the electron path. Numerical simulation are generally unavoidable to take into account the two dimensional projection of a three dimensional field.

It then gives an image of the magnetic induction flux perpendicular to the electron beam direction and projected over the beam path. An image of the projected components $B_{x}(\mathbf{r})=B_{x}(x, y)$ and $B_{y}(\mathbf{r})=B_{y}(x, y)$ of the magnetic induction can be calculated performing a partial derivative of $\phi(\mathbf{r})$ over $y$ and $x$ respectively

$$
\begin{aligned}
& \int B_{x}(\mathbf{r}) d z=-\frac{\hbar}{e} \frac{d}{d y} \phi(\mathbf{r}) \\
& \int B_{y}(\mathbf{r}) d z=\frac{\hbar}{e} \frac{d}{d x} \phi(\mathbf{r})
\end{aligned}
$$

\section{Micromagnetic and phase shift simulations}

Micromagnetic simulations are performed using the finite element package Magpar [S3] to simulate the entire write pole system using the dimensions of the write pole extracted from SEM images. We use the standard bulk values of the magnetic constants of the various materials comprising the write pole system (writer, shields, etc.) and generate the magnetic flux spreading in a tri-dimensional $(1 \mu \mathrm{m}, 1.5 \mu \mathrm{m}, 0.8 \mu \mathrm{m})$ box in front of the whole device for an applied current of $+40 \mathrm{~mA}$ (Fig. S3).

The resulting magnetic induction flux is used to calculate the phase shift of the electron beam passing across 
the box by integrating the simulated $B_{x}(x, y)$ and $B_{y}(x, y)$, over the thickness of the box and following Eq. S3.

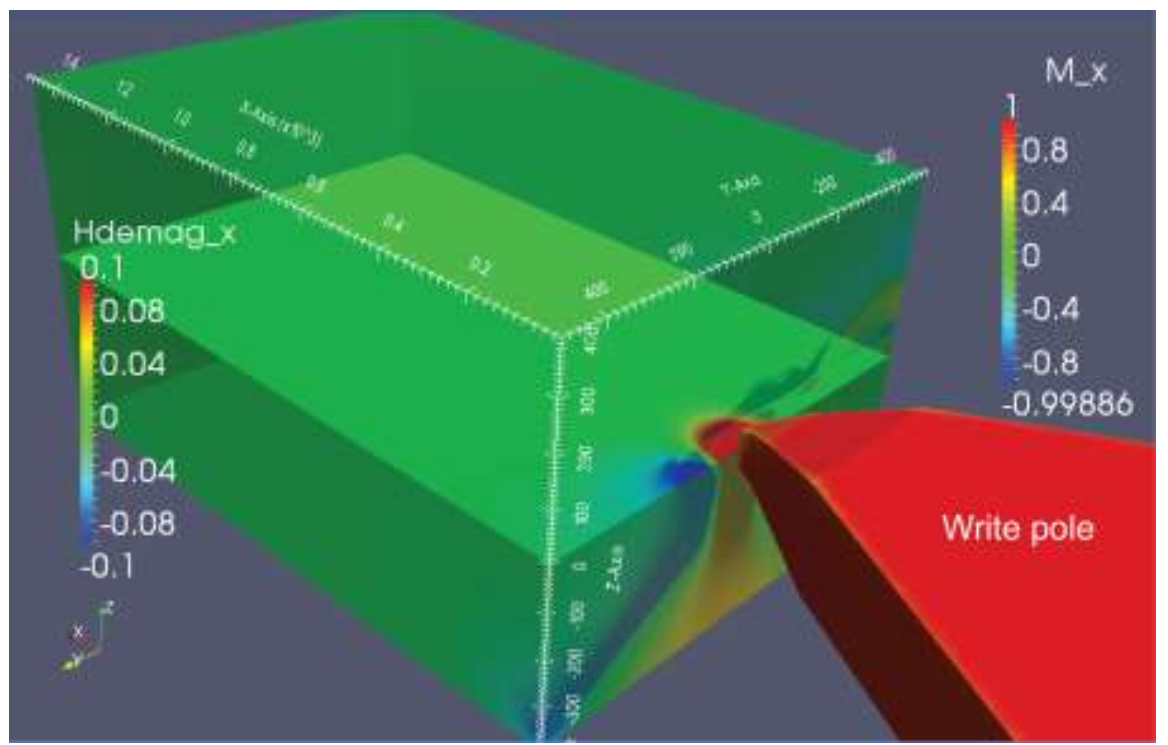

Figure $\mathbf{S 3}$ Box of $(1.5 \mu \mathrm{m}, 1 \mu \mathrm{m}, 0.8 \mu \mathrm{m})$ used for the micromagnetic simulations. The write pole is located at position $(0,0,0)$.

\section{References}

[S1] Aharonov, Y. \& Bohm, D. Significance of Electromagnetic Potentials in the Quantum Theory. Phys. Rev. 1959, 115, 485-491

[S2] Snoeck E. and Gatel C. "Magnetic Mapping Using Electron Holography" in Transmission Electron Microscopy in Micro-nanoelectronics ed. A. Claverie, Editeur : ISTE Ltd and John Wiley \& Sons Inc (2012)

[S3] Scholz, W. et al. Scalable parallel micromagnetic solvers for magnetic nanostructures. Comput. Mater. Sci. 2003, 28, 366-383. 